\title{
Increased expression of fatty acid synthase and acetyl-CoA carboxylase in the prefrontal cortex and cerebellum in the valproic acid model of autism
}

\author{
JIANLING CHEN ${ }^{1}$, WEI WU ${ }^{2}$, YINGMEI FU ${ }^{1}$, SHUNYING YU ${ }^{1}$, DONGHONG CUI ${ }^{1}$, \\ MIN ZHAO $^{1}$, YASONG DU ${ }^{1}$, JIJUN LI ${ }^{3}$ and XIAOHONG LI ${ }^{1}$ \\ ${ }^{1}$ Shanghai Key Laboratory of Psychotic Disorders, Shanghai Mental Health Center, \\ Shanghai Jiao Tong University School of Medicine, Shanghai 200030; ${ }^{2}$ Department of Pathology, Shanghai Pulmonary \\ Hospital, Tongji University, Shanghai 200433; ${ }^{3}$ Department of Integrative Medicine on Pediatrics, \\ Shanghai Children's Medical Center, Shanghai Jiao Tong University School of Medicine, Shanghai 200127, P.R. China
}

Received June 6, 2015; Accepted May 3, 2016

DOI: $10.3892 /$ etm.2016.3508

\begin{abstract}
The primary aim of the present study was to investigate alterations in enzymes associated with fatty acid synthesis, namely fatty acid synthase (FASN) and acetyl-CoA carboxylase (ACC), in the prefrontal cortex and cerebellum of the valproic acid (VPA)-induced animal model of autism. In this model, pregnant rats were given a single intraperitoneal injection of VPA, and prefrontal cortex and cerebellum samples from their pups were analyzed. The results of western blotting and reverse transcription-quantitative polymerase chain reaction analyses demonstrated that the protein and mRNA expression levels of FASN, ACC and phospho-ACC (pACC) were increased in the prefrontal cortex and cerebellum of the VPA model of autism. Furthermore, in the prefrontal cortex and cerebellum of the VPA model of autism, AMPK expression is increased, whereas PI3K and Akt expression are unchanged. This suggests that disorder of the phosphatidylinositol-4,5-bisphosphate 3-kinase (PI3K)/Akt/FASN and/or adenosine 5'-monophosphate-activated protein kinase (AMPK)/ACC pathway may be involved in the pathogenesis of autism. It is hypothesized that fatty acid synthesis participates in autism through PI3K/Akt/FASN and AMPK/ACC pathways.
\end{abstract}

Correspondence to: Dr Xiaohong Li, Shanghai Key Laboratory of Psychotic Disorders, Shanghai Mental Health Center, Shanghai Jiao Tong University School of Medicine, 600 Wanping Nan Road, Shanghai 200030, P.R. China

E-mail:1xh15@hotmail.com

Key words: fatty acid synthase, acetyl-CoA carboxylase, autism spectrum disorders, fatty acid, valproic acid

\section{Introduction}

Autism spectrum disorders (ASDs) are neurodevelopmental disorders with deficits in social interaction and social communication, in addition to stereotyped behaviors with restricted interests (1). Children with a mother who was exposed to anti-epileptic drug valproic acid (VPA) during pregnancy are more prone to have ASDs (2). As a result, a single administration of VPA to pregnant rats during the gestation period has been extensively used as an animal model of autism (3-5). Neuropathological studies suggest that autistic subjects have decreased numbers of cerebellar Purkinje cells, and impaired cortical migration (6). This indicates that cerebellum and cortex disorders may be involved in the VPA model of autism. There is also evidence of decreased levels of essential fatty acids in the red blood cells of autistic children and altered serum levels of polyunsaturated fatty acids (PUFAs) in the valproate-induced autism model $(7,8)$. Furthermore, Zhao et al detected reduced expression levels of associated liver metabolic enzymes $\Delta 5$-desaturase, $\Delta 6$-desaturase and elongase (Elov12) (7). These observations indicate that fatty acid and liver metabolic enzymes may be involved in the pathogenesis of autism.

When regarding the liver metabolic enzymes of fatty acids, it is important to take the process of fatty acid synthesis into consideration. Fatty acid synthesis involves the creation of fatty acids from acetyl-coenzyme A (CoA) and malonyl-CoA precursors through the catalytic action of fatty acid synthase (FASN). Acetyl-CoA can be transformed into malonyl-CoA by acetyl-CoA carboxylase (ACC), which is the rate-limiting enzyme during the fatty acid synthesis process. Researchers have shown that lipogenic enzymes, such as FASN and ACC, are highly expressed in the rodent brain during the early neonatal period and decline thereafter $(9,10)$. This suggests a neurodevelopmental role of FASN and ACC. Since ASDs are neurodevelopmental disorders, the aim of the present study was to detect the expression of FASN and ACC in the prefrontal cortex and cerebellum in the VPA-induced animal model of autism in order to determine the role of the fatty acid synthesis pathway in the pathogenesis of autism. 


\section{Materials and methods}

Animals and treatment. A total of 26 female (weight, 270-310 g) and 13 male (weight, 310-340 g) Sprague-Dawley rats were obtained from Laboratory Animal Research Center (Shanghai, China). The animals were maintained in a light- (lights on at 7:00 and lights off at 19:00), temperature- $\left(23 \pm 2^{\circ} \mathrm{C}\right)$ and humidity-controlled $(55 \pm 10 \%)$ environment and allowed $a d$ libitum food and water. The present study was performed in accordance with the guidelines of the National Institutes of Health (NIH) Guide for the Care and Use of Laboratory Animals (8th edition, 2011) and was approved by the Animal Care and Use Committee of Shanghai Jiao Tong University School of Medicine (Shanghai, China). The estrous cycle was monitored and the rats were mated overnight. The first day of gestation was considered to be when spermatozoa were found in the vaginal smear. Female rats in the experimental group were given a single intraperitoneal injection of VPA $(600 \mathrm{mg} / \mathrm{kg}$, $250 \mathrm{mg} / \mathrm{ml}$ ) on the 12.5 th day of pregnancy, whereas control females were given the same amount of physiological saline at the same time $(3,11)$. The offspring rats of the experimental females were marked as the VPA group, and of the control females were marked as the control group. Pups were anesthetized using sodium pentobarbital $(100 \mathrm{mg} / \mathrm{kg}$ body weight) and sacrificed by decapitation at postnatal day 7 . The brains were removed and prefrontal cortex and cerebellum samples were kept at $-80^{\circ} \mathrm{C}$ until the assays were performed.

Western blot analysis. Samples of the prefrontal cortex and cerebellum from the mouse brains were homogenized (1-2 min) in cold buffer $(10 \% \mathrm{w} / \mathrm{v})$ consisting of $50 \mathrm{mM}$ Tris- $\mathrm{HCl}(\mathrm{pH} 7.4)$, $8.5 \%$ sucrose, $2.0 \mathrm{mM}$ ethylenediamine tetraacetic acid, $10 \mathrm{mM} \beta$-mercaptoethanol and a protease inhibitor cocktail (Sigma-Aldrich, St. Louis, MO, USA). The protein concentration was quantified by the Bradford method using a protein assay kit from Bio-Rad Laboratories, Inc. (Hercules, CA, USA). The homogenate samples were mixed with $2 \mathrm{X}$ concentrated Laemmeli buffer (125 mM Tris-HCl, pH 6.8,4\% sodium dodecyl sulfate (SDS), $20 \%$ glycerol, $2 \% \beta$-mercaptoethanol, and $0.005 \%$ bromophenol blue) and separated by $8 \%$ SDS-polyacrylamide gel electrophoresis. The separated proteins were transferred onto a polyvinylidene fluoride membrane for $1 \mathrm{~h}$ at $100 \mathrm{~V}$ at $4^{\circ} \mathrm{C}$. The membranes were then incubated at room temperature for $1 \mathrm{~h}$ with blocking buffer, including $5 \%$ non-fat dry milk in Tris-buffered saline with $0.1 \%$ Tween 20 to block nonspecific reactions. After blocking, the blots were incubated with primary antibody overnight at $4^{\circ} \mathrm{C}$. The primary antibodies were rabbit antibodies targeting FASN (Gly46) (1:1,000; \#3180), ACC (1:1,000; \#3676), phospho (p)ACC (Ser 79) (1:1,000; \#11818), phosphatidylinositol-4,5-bisphosphate 3-kinase (PI3K p110; 1:1,000; \#4255), Akt (1:1,000; \#4691) and adenosine 5'-monophosphate-activated protein kinase (AMPK; 1:1,000; \#5831), acquired from Cell Signaling Technology, Inc. (Danvers, MA, USA), followed by an anti-rabbit IgG, horseradish peroxide-linked secondary antibody incubation for $1 \mathrm{~h}$ at room temperature (1:2,000; \#7074; Cell Signaling Technology, Inc.). $\beta$-Actin rabbit mAb (D6A8; \#8457) was used as a loading control. The immunoblots were developed by enhanced chemiluminescence according to the manufacturer's protocol (Merck Millipore, Darmstadt, Germany).
Reverse transcription-quantitative polymerase chain reaction (RT-qPCR) analysis. Total RNA was extracted from the cortex and cerebellum tissues using TRIzol reagent (Invitrogen; Thermo Fisher Scientific, Inc., Waltham, MA, USA) according to the manufacturer's protocol, and was used as the template for cDNA synthesis. All RNA preparation and handling steps were done under RNAse-free conditions. Then, $1 \mu \mathrm{g}$ total RNA from each sample were treated with DNase I (Fermentas; Thermo Fisher Scientific, Inc.), according to the manufacturer's instructions. Reverse transcription was performed using M-MLV. The following primer sets were used for qPCR: PI3K (sense 5'-AACACAGAAGACCAATACTC-3', anti-sense 5'-TTCGCC ATCTACCACTAC-3'), Akt (sense 5'-GTGGCAAGATGT GTATGAG-3', anti-sense 5'-CTGGCTGAGTAGGAGAAC-3'), AMPK (sense 5'-GACCTCGGTCAAGTGTCG-3', anti-sense 5'-TGGGTTATCAACGGGCTA-3'), ACC (sense 5'-CGCTGC GGTCAAGTGT-3', anti-sense 5'-CGTTGGCGTAGTTGT TATT-3'), FASN (sense 5'-ATGAAGAGGGACCATAAA-3', anti-sense 5'-ACAGGTGGGAACAAGG-3') and $\beta$-actin (sense 5'-CACCCGCGAGTACAACCTT-3', anti-sense 5'-CCCATA CCCACCATCACACC-3'). SYBR green-based qPCR assays were used for the gene expression analysis of PI3K, Akt, AMPK, ACC, FASN and the housekeeping gene $\beta$-actin. qPCR was performed using a Bio-Rad iQ5 Real-Time PCR system (Bio-Rad Laboratories, Inc.). The relative expression levels of the signaling molecules were calculated using the $2^{-\Delta \Delta C q}$ method with $\beta$-actin as the endogenous reference gene (12).

Statistical analysis. Data are presented as the mean \pm standard error of the mean. Blot densities were quantified using Image $\mathbf{J}$ software (National Institutes of Health, Bethesda, MD, USA). Comparisons between the VPA group and control group were performed by independent-samples t-test using IBM SPSS Statistics 20.0 software (IBM SPSS, Armonk, NY, USA). $\mathrm{P}<0.05$ was considered to indicate a statistically significant difference.

\section{Results}

FASN expression is increased in the prefrontal cortex and cerebellum in the VPA model of autism. To evaluate the expression changes of FASN in autism, a VPA model of autism was generated. Western blot analysis showed a 4-fold increase in FASN protein expression in the prefrontal cortex and a 2-fold increase in FASN expression in the cerebellum in the VPA group. The relative expression values (FASN/ $\beta$-actin) were $0.76 \pm 0.02$ for the VPA group and $0.17 \pm 0.01$ for the control group in the prefrontal cortex (Fig. 1), and $0.76 \pm 0.06$ for the VPA group and $0.33 \pm 0.03$ for the control group in the cerebellum (Fig. 2). Using RT-qPCR, it was detected that the mRNA level of FASN in the VPA group was 6.1-fold higher than that of the control group in the prefrontal cortex (Fig. 3) and 2.8-fold higher than that of the control group in the cerebellum (Fig. 4).

ACC expression is increased in the prefrontal cortex and cerebellum in the VPA model of autism. Western blot analysis was performed in order to evaluate the protein expression of ACC, the key enzyme of fatty acid synthesis. A significant increase in ACC expression was detected in the prefrontal 
A

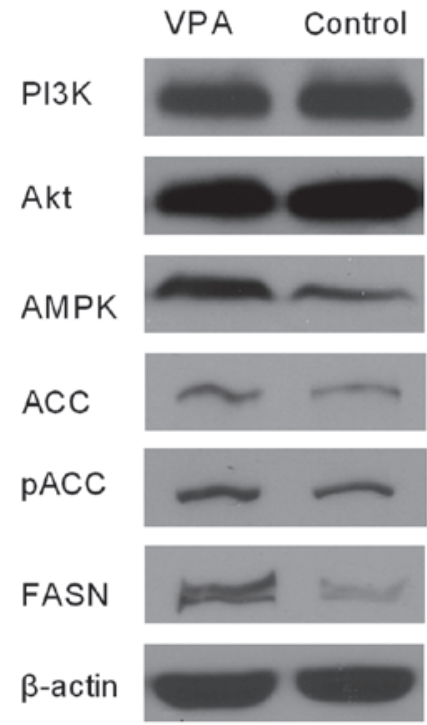

B

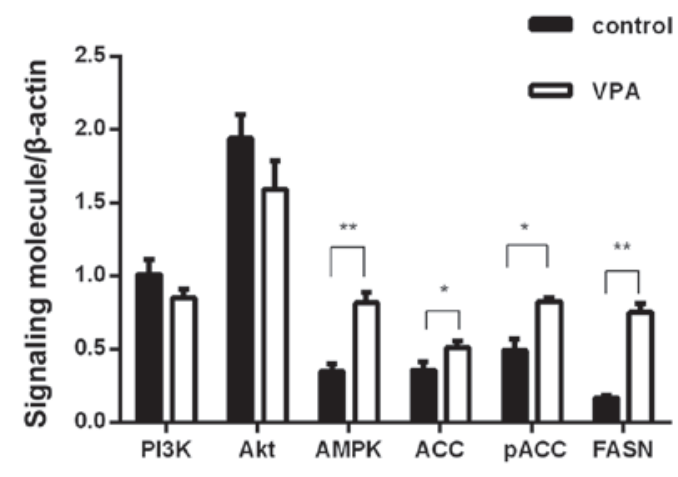

Figure 1. Signaling molecule protein expression in the prefrontal cortex of the valproic acid (VPA) model of autism. (A) Western blot of the prefrontal cortex in the VPA model of autism. (B) Quantification of the immunoblots following normalization using $\beta$-actin. Data are shown as the mean \pm standard error of the mean $(\mathrm{n}=8) .{ }^{*} \mathrm{P}<0.05,{ }^{* *} \mathrm{P}<0.01$ for the VPA group vs. the control. Significant differences between the VPA and control groups were identified by independent-samples t-test. ACC, acetyl-coenzyme A carboxylase; pACC, phospho-ACC; FASN, fatty acid synthase; AMPK, adenosine 5'-monophosphate-activated protein kinase; PI3K, phosphatidylinositol-4,5-bisphosphate 3-kinase.

A

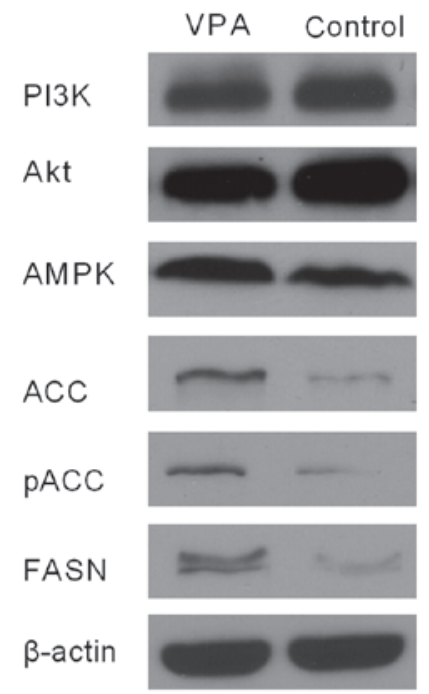

B

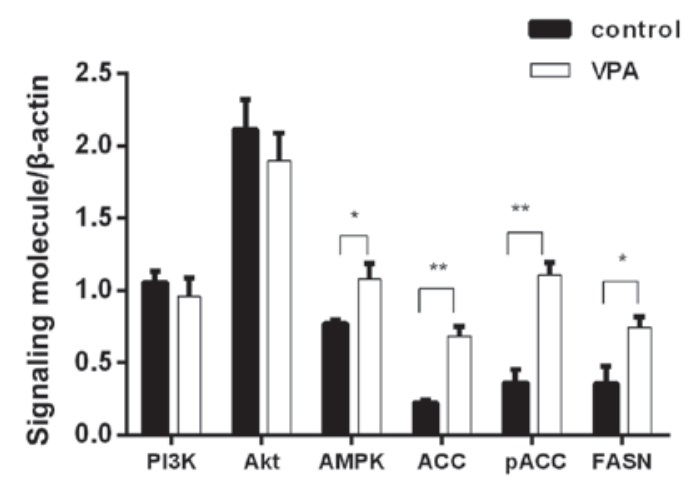

Figure 2. Signaling molecule protein expression in the cerebellum of the valproic acid (VPA) model of autism. (A) Western blot of the cerebellum in the VPA model of autism. (B) Quantification of the immunoblots following normalization using $\beta$-actin. The data are shown as the mean \pm standard error of the mean $(\mathrm{n}=8) .{ }^{*} \mathrm{P}<0.05,{ }^{* *} \mathrm{P}<0.01$ for the VPA group vs. the control. Significant differences between the VPA and control groups were identified by independent-samples t-test. ACC, acetyl-coenzyme A carboxylase; pACC, phospho-ACC; FASN, fatty acid synthase; AMPK, adenosine 5'-monophosphate-activated protein kinase; PI3K, phosphatidylinositol-4,5-bisphosphate 3-kinase.

cortex and cerebellum in the VPA model of autism. The relative expression values $(\mathrm{ACC} / \beta$-actin) were $0.52 \pm 0.02$ for the VPA group and $0.29 \pm 0.06$ for the control group in the prefrontal cortex (Fig. 1) and $0.64 \pm 0.03$ for the VPA group and $0.25 \pm 0.01$ for the control group in the cerebellum (Fig. 2). Using RT-qPCR, it was detected that the mRNA level of ACC in the VPA group was 1.7-fold higher than that of the control group in the prefrontal cortex (Fig. 3) and 4.3-fold higher than that of the control group in the cerebellum (Fig. 4).

pACC expression is increased in the prefrontal cortex and cerebellum in the VPA model of autism. To further elucidate the activity of ACC, the expression levels of pACC were detected. Phosphorylation by AMPK at Ser79 can inhibit the enzymatic activity of ACC (13). Compared with the control group, pACC expression in the prefrontal cortex and cerebellum in the VPA group was significantly upregulated. The relative expression levels (pACC $/ \beta$-actin) in the VPA and control groups were $0.94 \pm 0.04$ and $0.51 \pm 0.04$, respectively, in the prefrontal cortex (Fig. 1), and $1.34 \pm 0.13$ and $0.38 \pm 0.03$, respectively, in the cerebellum (Fig. 2).

AMPK expression is increased in the prefrontal cortex and cerebellum in the VPA model of autism. In order to investigate 


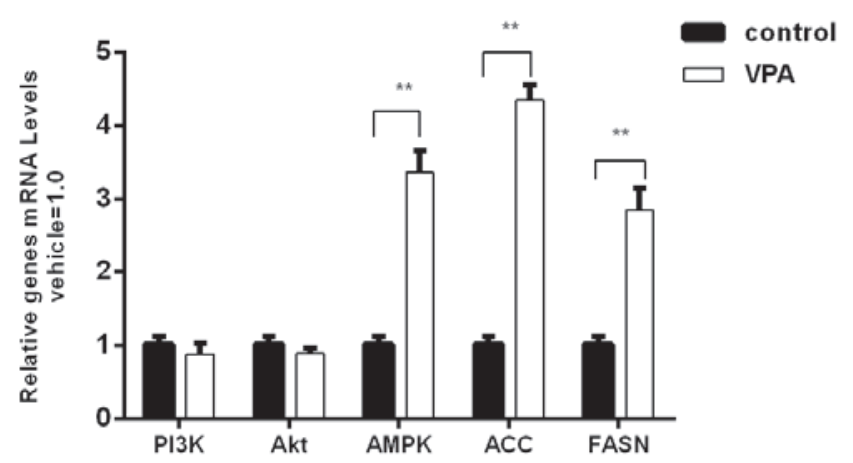

Figure 3. Signaling molecule mRNA expression levels in the prefrontal cortex of the valproic acid (VPA) model of autism. Relative expression levels of signaling molecules were determined by reverse transcription-quantitative polymerase chain reaction, and calculated using the $2^{-\Delta \Delta C q}$ method with $\beta$-actin as the endogenous reference gene. Data are shown as the mean \pm standard error of the mean $(\mathrm{n}=8) .{ }^{*} \mathrm{P}<0.05,{ }^{* * *} \mathrm{P}<0.01$ for the VPA group vs. the control. Significant differences between the VPA and control groups were identified by independent-samples t-test. ACC, acetyl-coenzyme A carboxylase; FASN fatty acid synthase; AMPK, adenosine 5'-monophosphate-activated protein kinase; PI3K, phosphatidylinositol-4,5-bisphosphate 3-kinase.

the role of AMPK in autism, the expression of AMPK was detected at the protein and mRNA levels. Western blot analysis showed that AMPK protein expression was significantly increased by $143.7 \%$ in the prefrontal cortex $(\mathrm{P}<0.01$; Fig. 1$)$ and by $36.7 \%$ in the cerebellum $(\mathrm{P}<0.05$; Fig. 2$)$ of the VPA-induced rats as compared with the controls. RT-qPCR analysis showed that the AMPK mRNA expression level in the VPA group was 3-fold higher than that in the control group in the prefrontal cortex (Fig. 3) and also in the cerebellum (Fig. 4).

PI3K and Akt expression are unchanged in the prefrontal cortex and cerebellum in the VPA model of autism. The expression of PI3K and Akt exhibited no significant differences between the VPA group and the control group at the protein and mRNA levels (Figs. 1-4). However, the protein and mRNA levels of PI3K and Akt in the VPA group manifested slightly reductions compared with the control group levels.

\section{Discussion}

The involvement of fatty acids in the pathogenesis of autism has previously been reported. Schultz et al found that children who were not breastfed or were fed infant formula without docosahexaenoic acid or arachidonic acid were more likely to have autism (14). Furthermore, Meguid et al reported that PUFA supplementation may play an important role in ameliorating the behavior of autistic children (15). Long chain acyl-CoA dehydrogenase (LCAD) is a key mitochondrial enzyme in the $\beta$-oxidation of branched chain and unsaturated fatty acids (16). Cox et al found that children with autism exhibit significant elevations of unsaturated fatty acid metabolites, in a similar manner to LCAD-deficient mice (17). Other metabolic abnormalities, including alterations of ammonia detoxification, reduced synthesis of $\omega-3$ docosahexaenoic acid, and abnormal cholesterol metabolism have also been detected in autistic children (18).

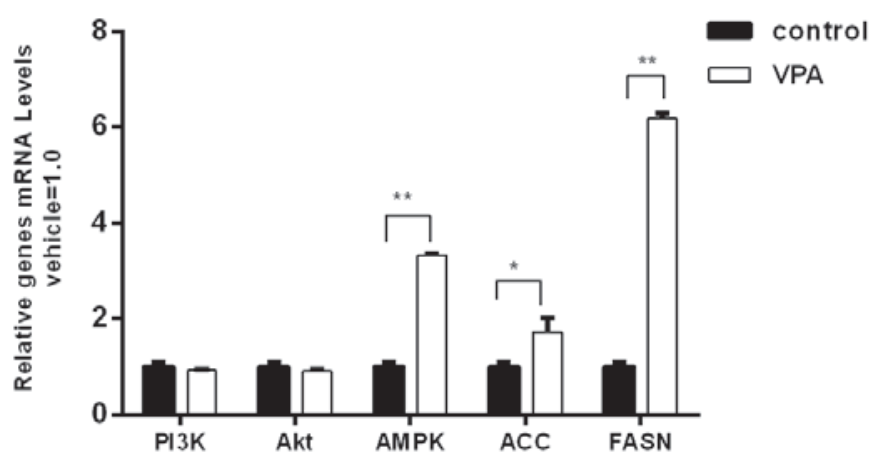

Figure 4. Signaling molecule mRNA expression in the cerebellum of the valproic acid (VPA) model of autism. Relative expression levels of signaling molecules were determined by reverse transcription-quantitative polymerase chain reaction, and calculated using the $2^{-\Delta \Delta \mathrm{Cq}}$ method with $\beta$-actin as the endogenous reference gene. Data are shown as the mean \pm standard error of the mean $(n=8) .{ }^{*} \mathrm{P}<0.05,{ }^{* *} \mathrm{P}<0.01$ for the VPA group vs. the control. Significant differences between the VPA and control groups were identified by independent-samples t-test. ACC, acetyl-coenzyme A carboxylase; FASN, fatty acid synthase; AMPK, adenosine 5'-monophosphate-activated protein kinase; PI3K, phosphatidylinositol-4,5-bisphosphate 3-kinase.

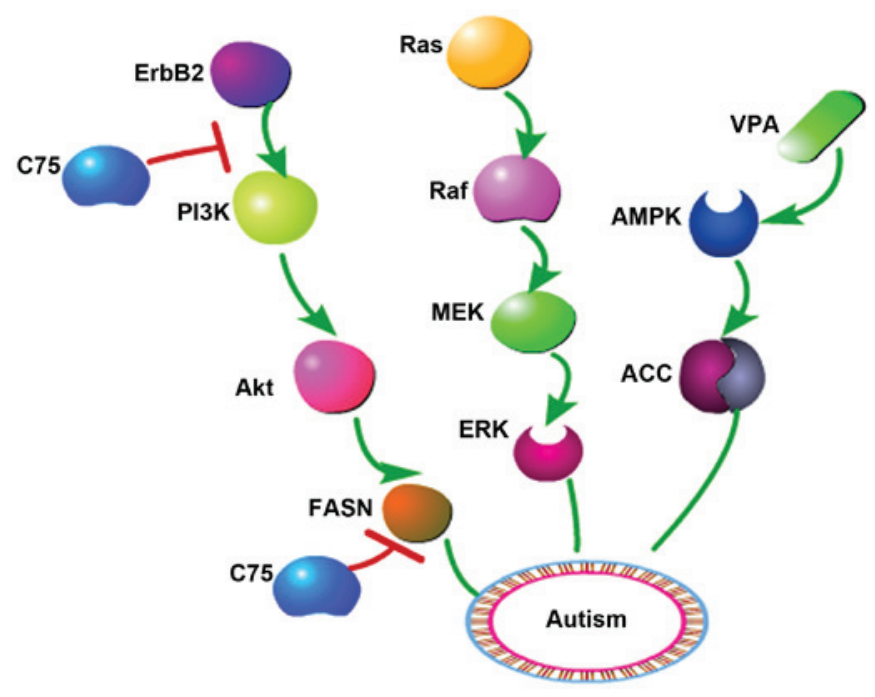

Figure 5. Summary of the pathways associated with autism. The red arrows representinhibition, and the green arrows represent activation. ACC, acetyl-coenzyme A carboxylase; AMPK, adenosine 5'-monophosphate-activated protein kinase; ERK, extracellular signal-regulated kinase; FASN, fatty acid synthase; MEK, mitogen-activated protein kinase kinase; PI3K, phosphatidylinositol-4,5-bisphosphate 3-kinase; VPA, valproic acid.

FASN is an enzyme encoded by the $F A S N$ gene in humans. Its main function is to catalyze the synthesis of palmitate from acetyl-CoA and malonyl-CoA in the presence of nicotinamide adenine dinucleotide (NADPH), and into long-chain saturated fatty acids (19). Furthermore, Veigel et al reported that FASN is a metabolic marker of cell proliferation (20). The results of the present study show that the protein expression of FASN is increased in the prefrontal cortex and cerebellum in the VPA model of autism. The upregulation of FASN suggests that cell proliferation is increased in autism. Consistently, increased progenitor proliferation and premature cell cycle withdrawal, which occur as a consequence of alterations affecting the Ras/Raf/MEK extracellular signal-regulated kinase (ERK) pathway, have been observed in a 16p11.2 deletion murine 
model of autism (21), as shown in Fig. 5. Previous studies by the authors of the present study have also indicated that upregulation of the Ras/Raf/MEK/ERK pathway may be involved in autism $(22,23)$. In addition, enhanced progenitor proliferation and cell cycle exit can lead to premature depletion of progenitor pools and alteration of the number and frequency of cortical neurons (21). FASN catalyzes the formation of phospholipids for membrane microdomains that accommodate receptor tyrosine kinases, including ErbB1 and ErbB2. The FASN inhibitor C75 can repress the expression of FASN, ErbB1, ErbB2 and Akt, suggesting that FASN may participate in the ErbB/Akt pathway (24). The ErbB2 inhibitor lapatinib is able to suppress the activity of the ErbB2/PI3K/Akt/FASN pathway (25). Dysregulation of the PI3K/Akt pathway has been shown to have an association with autism (26); therefore, it is speculated that alterations of the ErbB2/PI3K/Akt/FASN signaling pathway may be involved in autism.

ACC catalyzes the irreversible carboxylation of acetyl-CoA to produce malonyl-CoA, a building block for new fatty acids (27). Malonyl-CoA is also able to inhibit the combination of fatty acids and carnitine to prevent fatty acids from entering the mitochondria for oxidation and degradation (28). Reduced NADH oxidase activity in lymphocytic mitochondria has been detected in children with autism (29). Elevated plasma pyruvate levels, increased mitochondrial rates of hydrogen peroxide production and mitochondrial DNA over-replication have been observed in autistic subjects (29). In addition, there have been studies demonstrating elevations in short-chain and long-chain acyl-carnitines in propionic acid-induced rodent autism models $(30,31)$. ACC can be phosphorylated and inactivated at Ser79 by AMPK (13). VPA is an activator of AMPK (32). The results of the present study indicate that the intraperitoneal injection of VPA increased the protein expression of ACC, which may suggest upregulation of the AMPK/ACC pathway in the VPA animal model of autism. The AMPK/ACC pathway regulates cellular survival or apoptosis and energy homeostasis in the hypothalamus (33). Therefore, it is speculated that an apoptosis pathway may be involved in the development of autism. At present, the clinical treatment of autism remains a big challenge. Researchers have demonstrated that a ketogenic diet (KD) plays a potential therapeutic role and may attenuate social and metabolic alterations identified in the VPA model of autism (34). Furthermore, the treatment effect of the KD may be caused by a reduction in the activity of AMPK/ACC pathway and heat shock protein 70 to decrease kainic acid-induced hippocampal cell death (35).

In this context, the results of the present study reveal important alterations in the protein expression levels of FASN and ACC in the prefrontal cortex and cerebellum in the VPA model of autism. These results suggest that ErbB2/PI3K/Akt/ FASN and/or AMPK/ACC pathway disorders are likely to be involved in the induction of autistic symptoms. Therefore, it is hypothesized that fatty acid synthesis may participate in autism through ErbB2/PI3K/Akt/FASN and AMPK/ACC pathways.

\section{Acknowledgements}

This study was supported by funding for an 'oriental scholar' distinguished professor from the Shanghai Mental Health
Center, Shanghai Jiao Tong University School of Medicine and the National Nature Science Foundation of China (grant nos. 81371491 and 8152803).

\section{References}

1. American Psychiatric Association: Diagnostic and Statistical Manual of Mental Disorders (DSM-5). 5th edition. American Psychiatric Publishing, Arlington, VA, 2013.

2. Ornoy A: Valproic acid in pregnancy: How much are we endangering the embryo and fetus? Reprod Toxicol 28: 1-10, 2009.

3. Rodier PM, Ingram JL, Tisdale B and Croog VJ: Linking etiologies in humans and animal models: Studies of autism. Reprod Toxicol 11: 417-422, 1997.

4. Chomiak T and Hu B: Alterations of neocortical development and maturation in autism: Insight from valproic acid exposure and animal models of autism. Neurotoxicol Teratol 36: 57-66, 2013.

5. Foley AG, Gannon S, Rombach-Mullan N, Prendergast A, Barry C, Cassidy AW and Regan CM: Class I histone deacetylase inhibition ameliorates social cognition and cell adhesion molecule plasticity deficits in a rodent model of autism spectrum disorder. Neuropharmacology 63: 750-760, 2012.

6. Bauman ML: Microscopic neuroanatomic abnormalities in autism. Pediatrics 87: 791-796, 1991.

7. Zhao G, Gao J, Liang S, Wang X, Sun C, Xia W, Hao Y, Li X Cao Y and Wu L: Study of the serum levels of polyunsaturated fatty acids and the expression of related liver metabolic enzymes in a rat valproate-induced autism model. Int J Dev Neurosci 44: $14-21,2015$

8. Brigandi SA, Shao H, Qian SY, Shen Y, Wu BL and Kang JX: Autistic children exhibit decreased levels of essential fatty acids in red blood cells. Int J Mol Sci 16: 10061-10076, 2015.

9. Saito M, Chakraborty G, Mao RF and Vadasz C: Developmental profiles of lipogenic enzymes and their regulators in the neonatal mouse brain. Neurochem Res 34: 1945-1954, 2009.

10. Spencer EB, Bianchi A, Widmer J and Witters LA: Brain acetyl-CoA carboxylase: Isozymic identification and studies of its regulation during development and altered nutrition. Biochem Biophys Res Commun 192: 820-825, 1993.

11. Schneider T and Przewłocki R: Behavioral alterations in rats prenatally exposed to valproic acid: Animal model of autism. Neuropsychopharmacology 30: 80-89, 2005.

12. Livak KJ and Schmittgen TD: Analysis of relative gene expression data using real-time quantitative PCR and the 2(-Delta Delta C(T)) Method. Methods 25: 402-408, 2001.

13. Ha J, Daniel S, Broyles SS and Kim KH: Critical phosphorylation sites for acetyl-CoA carboxylase activity. J Biol Chem 269: 22162-22168, 1994.

14. Schultz ST, Klonoff-Cohen HS, Wingard DL, Akshoomoff NA, Macera CA, Ji M and Bacher C: Breastfeeding, infant formula supplementation and autistic disorder: The results of a parent survey. Int Breastfeed J 1: 16, 2006.

15. Meguid NA, Atta HM, Gouda AS and Khalil RO: Role of polyunsaturated fatty acids in the management of Egyptian children with autism. Clin Biochem 41: 1044-1048, 2008.

16. Lea W, Abbas AS, Sprecher H, Vockley J and Schulz H: Long-chain acyl-CoA dehydrogenase is a key enzyme in the mitochondrial beta-oxidation of unsaturated fatty acids. Biochim Biophys Acta 1485: 121-128, 2000.

17. Cox KB, Hamm DA, Millington DS, Matern D, Vockley J, Rinaldo P, Pinkert CA, Rhead WJ, Lindsey JR and Wood PA: Gestational, pathologic and biochemical differences between very long-chain acyl-CoA dehydrogenase deficiency and long-chain acyl-CoA dehydrogenase deficiency in the mouse. Hum Mol Genet 10: 2069-2077, 2001.

18. Clark-Taylor T and Clark-Taylor BE: Is autism a disorder of fatty acid metabolism? Possible dysfunction of mitochondrial beta-oxidation by long chain acyl-CoA dehydrogenase. Med Hypotheses 62: 970-975, 2004.

19. PerssonB,Kallberg Y,Bray JE,BrufordE,DellaportaSL,Favia AD, Duarte RG, Jörnvall H, Kavanagh KL, Kedishvili N, et al: The SDR (short-chain dehydrogenase/reductase and related enzymes) nomenclature initiative. Chem Biol Interact 178: 94-98, 2009.

20. Veigel D, Wagner R, Stübiger G, Wuczkowski M, Filipits M, Horvat R, Benhamú B, López-Rodríguez ML, Leisser A, Valent $\mathrm{P}$, et al: Fatty acid synthase is a metabolic marker of cell proliferation rather than malignancy in ovarian cancer and its precursor cells. Int J Cancer 136: 2078-2090, 2015. 
21. Pucilowska J, Vithayathil J, Tavares EJ, Kelly C, Karlo JC and Landreth GE: The 16p11.2 deletion mouse model of autism exhibits altered cortical progenitor proliferation and brain cytoarchitecture linked to the ERK MAPK pathway. J Neurosci 35: 3190-3200, 2015.

22. Yang K, Cao F, Sheikh AM, Malik M, Wen G, Wei H, Brown WT and $\mathrm{Li} \mathrm{X.:} \mathrm{Up-regulation} \mathrm{of} \mathrm{Ras/Raf/ERK1/2} \mathrm{signaling} \mathrm{impairs}$ cultured neuronal cell migration, neurogenesis, synapse formation, and dendritic spine development. Brain Struct Funct 218: 669-682, 2013.

23. CaoFJ,Zhang X,Liu T,LiXW,Malik Mand Feng SQ: Up-regulation of Ras/Raf/ERK1/2 signaling in the spinal cord impairs neural cell migration, neurogenesis, synapse formation, and dendritic spine development. Chin Med J (Engl) 126: 3879-3885, 2013.

24. Grunt TW, Wagner R, Grusch M, Berger W, Singer CF, Marian B, Zielinski $\mathrm{CC}$ and Lupu R: Interaction between fatty acid synthase- and ErbB-systems in ovarian cancer cells. Biochem Biophys Res Commun 385: 454-459, 2009.

25. Long XH, Zhang GM, Peng AF, Luo QF, Zhang L, Wen HC, Zhou RP, Gao S, Zhou Y and Liu ZL: Lapatinib alters the malignant phenotype of osteosarcoma cells via downregulation of the activity of the HER2-PI3K/AKT-FASN axis in vitro. Oncol Rep 31: 328-334, 2014.

26. Chen J, Alberts I and Li X: Dysregulation of the IGF-I/PI3K/ $\mathrm{AKT} / \mathrm{mTOR}$ signaling pathway in autism spectrum disorders. Int J Dev Neurosci 35: 35-41, 2014.

27. Abu-Elheiga L, Matzuk MM, Abo-Hashema KA and Wakil SJ: Continuous fatty acid oxidation and reduced fat storage in mice lacking acetyl-CoA carboxylase 2. Science 291: 2613-2616, 2001.

28. Nelson DL and Cox MM: Lehninger Principles of Biochemistry. 5th edition. W. H. Freeman and Company, London, p806, 2008.
29. Giulivi C, Zhang YF, Omanska-Klusek A, Ross-Inta C, Wong S, Hertz-Picciotto I, Tassone F and Pessah IN: Mitochondrial dysfunction in autism. JAMA 304: 2389-2396, 2010.

30. Frye RE, Melnyk S and Macfabe DF: Unique acyl-carnitine profiles are potential biomarkers for acquired mitochondrial disease in autism spectrum disorder. Transl Psychiatry 3: e220, 2013.

31. Thomas RH, Foley KA, Mepham JR, Tichenoff LJ, Possmayer F and MacFabe DF: Altered brain phospholipid and acylcarnitine profiles in propionic acid infused rodents: Further development of a potential model of autism spectrum disorders. J Neurochem 113: 515-529, 2010.

32. Avery LB and Bumpus NN: Valproic acid is a novel activator of AMP-activated protein kinase and decreases liver mass, hepatic fat accumulation and serum glucose in obese mice. Mol Pharmacol 85: 1-10, 2014.

33. Wen JP, Liu CE, Hu YT, Chen G and Lin LX: Globular adiponectin regulates energy homeostasis through AMP-activated protein kinase-acetyl-CoA carboxylase (AMPK/ACC) pathway in the hypothalamus. Mol Cell Biochem 344: 109-115, 2010.

34. Ahn Y, Narous M, Tobias R, Rho JM and Mychasiuk R: The ketogenic diet modifies social and metabolic alterations identified in the prenatal valproic acid model of autism spectrum disorder. Dev Neurosci 36: 371-380, 2014.

35. Jeon BT, Lee DH, Kim KH, Kim HJ, Kang SS, Cho GJ, Choi WS and Roh GS: Ketogenic diet attenuates kainic acid-induced hippocampal cell death by decreasing AMPK/ACC pathway activity and HSP70. Neurosci Lett 453: 49-53, 2009. 